\title{
EDITORIAL
}

\section{Pulmonary Therapy Podcast-COVID-19: Research and Real-World Experiences from the Editorial Board}

Kai Michael Beeh · Nazia Chaudhuri · Timothy J. Craig •

Alan Kaplan · Marcus Peter Kennedy

Received: April 13, 2021 / Accepted: April 15, 2021 / Published online: May 11, 2021

(C) The Author(s) 2021

\section{ABSTRACT}

The Editorial Board have prepared a podcast describing their experiences over the past year of the COVID-19 pandemic. The Editorial Board describe how COVID-19 impacted their research and how the initial clinical response changed over the course of the year in terms of treatment, personal protective equipment (PPE), and policy changes. The podcast and transcript can

Supplementary Information The online version contains supplementary material available at https:// doi.org/10.1007/s41030-021-00157-6.

K. M. Beeh ( $\square)$

INSAF Respiratory Research Institute, Wiesbaden, Germany

e-mail: k.beeh@insaf-wi.de

N. Chaudhuri

North West Interstitial Lung Disease Unit,

University Hospital of South Manchester,

Wythenshawe Hospital, Manchester, UK

N. Chaudhuri

Manchester Academic Health Science Centre, The

University of Manchester, Manchester, UK

T. J. Craig

Department of Medicine and Pediatrics, Penn State

University, State College, PA, USA

\section{A. Kaplan}

Department of Family and Community Medicine,

University of Toronto, Toronto, Canada be viewed below the abstract of the online version of the manuscript. Alternatively, the podcast and transcript can be downloaded here: https://doi.org/10.6084/m9.figshare.14402291

Keywords: COVID-19; Podcast; Pulmonary; Respiratory; Vaccine

\section{INTRODUCTION}

Hello, and thank you for listening to the Pulmonary Therapy podcast on COVID-19-

\author{
A. Kaplan \\ Primary Care Respiratory Research, Observational \\ and Pragmatic Research Institute, Singapore, \\ Singapore
A. Kaplan
Family Physician Airways Group of Canada,
Toronto, Canada \\ M. P. Kennedy \\ Department of Respiratory Medicine, Cork \\ University Hospital, Wilton, Cork, Republic of \\ Ireland \\ M. P. Kennedy \\ University College Cork, Cork, Ireland
}


Research and Real-World Experiences from the Editorial Board. My name is Christopher Vautrinot and I am the managing editor of the journal. Together with a number of the journal's Editorial Board members, we have compiled clips, submitted by the board, who describe their experience over the past year of the COVID-19 pandemic. The Editorial Board describe how COVID-19 impacted their research and how the initial clinical response changed over the course of the year in terms of treatment, PPE [personal protective equipment] and policy changes. We hope you find the podcast informative and useful.

\section{KAI MICHAEL BEEH}

Hi, everybody. My name is Kai Michael Beeh. I am a pulmonologist from Germany in Wiesbaden. I am the medical director of the insaf Respiratory Research Institute and I also lead a private pulmonology practice. I am one of the editors of the journal Pulmonary Therapy.

So, can you tell us how COVID has impacted your research? COVID had quite a striking impact on my work both as a clinician but also as a researcher. Now, as you may know, in my institute we do a lot of clinical trials in obstructive airway diseases, namely asthma and COPD, so there was a lot of that work that was delayed, put on hold, or even sometimes abruptly discontinued due to the outbreak of the pandemic. So there was a great deal of uncertainty and also quite a period where we actually totally had to adjust our organisation of research there, taken together with the fact that many patients were quite insecure when the pandemic started: how to behave, how they would continue with their visits and so on. So it was quite a big interruption of our normal work.

Second question, how did COVID impact your clinic during the initial outbreak? Well, there were a lot of measures that had to be installed during the initial outbreak. First of all, we reduced measurements with the potential of aerosol generation like forced spirometry, particularly airway challenge and sputum inductions, stuff like that. We were starting to equip staff, but also ourselves of course, with personal protective equipment, and after a while we got used to this new situation, and then of course with the precautions, and also some evidence coming up that certain measures could be installed to actually minimise the risk of infection, or spread of COVID would be effective. After this came through we were more and more confident to perform measurements also with our patients on site. So currently we are in a situation where we are doing most things quite regularly and quite normally but under, of course, a strict regime of precautionary measures.

The third question, what lessons have been learned so far? Well, both as a researcher and as a clinician, the most positive lesson of the last year was the rapid development of a very effective vaccine, in fact, different very effective vaccines. I think that was really a type of a best way or best world scenario that this happened so fast, and we were able to roll out vaccines already at the end of 2020. Although of course the logistical challenges seem to be far more important than the medical aspects of vaccination, but that's something, of course another lesson that we need to learn. What I found quite disappointing was the relatively poor outcome of actually the COVID treatments, so the clinical trials performed in a number of agents that provided some hope of efficacy versus COVID19. They have been largely disappointing, and also the quality of research in total sometimes was quite disappointing. So there were many studies with few patients, quite often underpowered, not properly enrolling, not properly organised and also structured clinical trial protocols. So I think it's very important, a lesson to be learned is that we need to be better prepared and we need to have protocols and platforms installed in case of pandemics.

\section{NAZIA CHAUDHURI}

Hi. My name's Nazia Chaudhuri. I'm an interstitial lung disease specialist in Manchester in the United Kingdom. I am also a research lead for interstitial lung disease in Manchester.

So one of the first questions is how has COVID impacted my research? Well, during the 
first wave in March, we were very much all redeployed to the wards because of the sheer number of patients on our wards. So initially there was a massive impact on our research. All our research, particularly in idiopathic pulmonary fibrosis, was put on hold because all our research staff were also redeployed to the wards. This has had a massive impact obviously on the access of research trials for our patients, but similarly, our patients were also shielding and were told to stay away from the hospital, and stay safe, and stay at home. So our patients also weren't very keen on coming to the hospital for their research trials. Our focus very much shifted to the RECOVERY trial and delivering COVID-related research, and certainly Manchester is one of the highest recruiters in COVIDrelated research in the United Kingdom. But as we came out of the first wave, even though we are now into our second wave, thankfully, the impact on research hasn't been as great. So, research for patient needs, particularly related to idiopathic pulmonary fibrosis where our patients don't have any other treatment options, is allowed to continue. So we are certainly continuing to recruit to the GALACTIC trial and we have a number of patients who have recently been screened and recruited to that. Unfortunately, the GALAPOGAS trial was put on hold. So hopefully we are hoping to get back on track with our research and invite our patients, in a safe environment, to continue with regards to access to research trials.

So the next question is, how did COVID impact my clinic during the initial outbreak? Well, again, unfortunately, in the first wave all our clinics were cancelled for the first few months, just because the sheer number of patients we had as inpatients on the wards in March. But, thankfully, from July onwards we have been able to reinstate our interstitial lung disease clinics, but we've reinstated them in different ways. We are certainly working very differently now during this COVID pandemic. All our clinics are now virtual. We are using virtual platforms were we can phone our patients with video consultations and assess our patients virtually-negating the need to bring our patients to big, busy waiting rooms. In Manchester we have approximately ten clinic rooms going on at the same time in a small confined area in the morning and the afternoon, and you can imagine that would be in excess of 100 patients in the morning and afternoon, and certainly we can't socially distance. So most of our clinics are now virtual. We are inviting patients we are concerned about for face-to-face consultations. COVID had a massive impact on our lung function capacity. Again, due to the AGP (aerosol-generating procedures), we had to have increased time between our lung function procedures, and so that really reduced our capacity. We've used innovative ways of increasing our lung function capacity by working out of different areas around the trust. Providing lung function and also providing lung function in community hospitals so that patients can come elsewhere for their lung function, and slowly but surely, as we come out of the pandemic, we are increasing our capacity. Obviously we are all wearing full PPE in our clinics with masks and gloves and aprons, and we are very much socially distancing our patients, so that we are timing their attendance to clinics so that there is minimal contact with other people.

So the next question is, how has this initial reaction to the pandemic developed over time and what lessons have been learnt? Well certainly, thankfully, for interstitial lung disease, one of our key thoughts was to continue our access to treatments for our patients who have debilitating symptoms as well as bad prognosis, for example our patients with idiopathic pulmonary fibrosis. So we have maintained our access to antifibrotics by performing virtual clinics. By relying on our local physicians to make sure that they've done all the lung function and blood tests so the patient is ready when they come to see us. We are performing a one-stop shop for our patients, particularly for our new patients. We are able to perform our new patient assessments in a non-COVID area in our hospital so patients can come and feel safe. They can feel safe that they are away from our COVID areas. We adhere to full PPE procedures with hand gel and masks so our patients feel safe, and so we have learnt that we can maintain our outpatient practice as much as possible during this pandemic, and certainly we 
have not cancelled all our outpatient activity, and tried to resume and very much have tried to catch up with our outpatient activity. For interstitial lung disease, thankfully, there have been some policy changes. So, for example, in the UK, you always had to have an up-to-date lung function for prescribing antifibrotics, because we have a restriction with an FVC [forced vital capacity] of $50-80 \%$, and thankfully NHS England agreed that as long as that lung function was in 12 months that we could use historic lung function, and that was really to the benefit of our patients, to ensure they got timely access to treatments, particularly antifibrotics. We perform a lot of virtual monitoring clinics, so our pharmacists and our specialist nurses perform a lot of virtual monitoring clinics so that we can maintain safe prescribing of immunosuppressants such as mycophenolate and our antifibrotics. So, all in all, the COVID pandemic has been very challenging but I think we have worked our way through. We have provided our patients, on an outpatient perspective, safe care so that they can have continued care in our interstitial lung disease service. We are now seeing light at the end of the tunnel, and hopefully with the vaccination rollout, we will see the case numbers of COVID in the community decrease so our patients feel confident that they can come to the hospital for their clinic appointments. But certainly COVID will change our future way of working, and we will work more virtually in the future, as well as with our patient, but also from an educational perspective as well.

\section{TIMOTHY J CRAIG}

So, hello. I'm Dr. Timothy Craig. I'm from Penn State University. which is in Pennsylvania, USA. I'm an allergist immunologist in a pulmonary group. I focus on rare diseases: alpha- 1 antitrypsin, hereditary angioedema and common variable immunodeficiency. In addition, the majority of my work, despite specializing in orphan diseases, is actually in asthma. I've been doing this for 25 years. We have a large section of allergy in the pulmonary group and I think we provide really good services.
So, one of the questions that came up was how did COVID affect our research? So, you know, it's been strange over the year here-I guess, a year and a few months. So, at first, everything shut down, but then what happened, we were able to start recruiting patients for clinical trials phase 2 and 3s. And, actually, I thought it worked out well because a lot of patients actually had free time, they didn't have to go to work or they were working from home, and so it was easy to recruit patients to come into our clinical trials. Unfortunately, any other trial where there wasn't any benefit to the patients such as phase $4 \mathrm{~s}$ and some, just laboratory collection, went poorly, and we had to cease recruitment for those studies and actually had to stop them. In addition, we were doing some basic research work on a microRNA, and unfortunately, we had to stop that too; however, we finally got that going about 3-4 months ago. So, right now, we're back up, and our basic research also, and we've continued for clinical research. And lastly, for the phase $4 \mathrm{~s}$ and other studies that really had no benefit to a patient, we've been able to start them about a month ago. So, in some ways, it impacted us, but in some ways, that actually worked out well for us.

You know, similarly for clinic, you know, how it impacted our clinic, we went $100 \%$ virtual up first. Unfortunately, with that we had to stop doing pulmonary function tests and almost all our procedures. And with that, our income drops significantly, and the amount of patients that we saw stayed. It's just that the reimbursements for telehealth was much less. However, we did really well. We switched to a platform, an official platform, called Amwell, and so actually, almost 100\%-I shouldn't sayI think it was $95 \%$ were actually on a telehealth platform, which allowed for better reimbursement than just a phone conversation with the patient. So I think we were, we did good on that conversion.

But then we started opening up, once we got over the first wave, and we opened up, and we decided to do 50/50-50\% clinic and 50\% telehealth-so that we could have more procedures. And that's worked out well. My no-show rate's been minimal, but my colleagues had a fair 
amount of no shows, and in fact, if you looked at the data, there was less no shows for telehealth than there was in face-to-face clinic. So, we're still now-even though we're not in another wave, but even between these two waves, or surge, whatever you want to refer to it as-we still are 50/50, because those statistics demonstrate in that people were more likely to show up for a telehealth visit. So, it's been an interesting time. I personally prefer face-to-face, and I am going to be moving in that direction as fast as I can. Probably by July, I'll do all face-toface. But I know my colleagues, some of them enjoy telehealth more, and they'll probably still stay in that-that 50/50 ratio.

One of the things with this, though, is that we still haven't got spirometry and some of the other testing we do-exhaled nitric oxide-up, so because of not having the proper filters and our spirometer and not having a room that is negative pressure. So that's going to be, that is a large part of our income, and we'll have to some way find a way to modify that. Not only is it income, though, for following COPD and asthma patients without spirometry is for me very difficult. So, you know, I think that how we've really kind of emerged from this is, you know, from the beginning of the pandemic to now is that we really gathered confidence that we can do telehealth. And I feel good about it and feel that we're doing a good job. So that's pretty rewarding. It has, however, impacted our bottom line, which is, you know, in a university, that's very important. We have to support our employees and everything else. But I think the majority of us now that we're vaccinated at least feel a lot more confident, you know, moving back into the face-to-face visits bymaybe that's a false sense of security in some ways, but, you know, it's nice too to be able to have some face-to-face meetings. It's nice to be able to drink a cup of coffee in my office, which is a shared office, the clinical office at least. And I think that my research, nurses, it's really been a nice building up confidence for them now that they're vaccinated and the same above clinic nurses. Unfortunately, not all our clinic nurses have got vaccinated, but our policy in the hospital, much to my dismay, is that you can't ask anybody if they've been vaccinated because of a HIPAA violation.

But I do think that if we continue to vaccinate like the federal government and United States wants us to, that come this summer it may be very appropriate to start doing everything we have in the past done before the pandemic. So, good opportunity, good learning. So, yeah, for our patients we've lost, we lost quite a few here, mainly more family and friends than actually my patients. But from everything bad, comes some good points. With that, you know, I wish you all good luck, and please be safe out there. And I hope that these times, even though they've been trying, that you gain some fortune from it. And I guess with fortune, is sounds like more a gain, but you've at least obtained some information to better your life, and like you do in your clinic and your research. So, thank you for this opportunity, and have a great day.

\section{ALAN KAPLAN}

Hello. My name is Dr. Alan Kaplan, a family physician from Toronto, Canada. I'm the chair of the Family Physician Airways Group of Canada, a vice president of the Respiratory Effectiveness Group and an honorary professor of primary care research of the Observational \& Pragmatic Research Institute in Singapore.

How has COVID impacted my research? Well, clearly, in-person studies are on hold. Virtual assessments, however, do allow surveys and behavioural research. Initially the issues are mostly of COVID on respiratory issues. I think our next big issue is going to be in primary care, but how we deal with long haulers.

How did COVID impact my clinic during the initial outbreak? Well, clearly, we had to have screening done before anyone was actually seen, although most things were done virtually initially, and the government did step up to create virtual fee codes. We used masks and goggles and handwashing for low risk, moving up to PPE and N95 for people with high risk. More recently we've reinstituted spirometry testing with screening done first, the rooms cleaned post-op and PPE for the staff as well. 
How has this initial reaction to the pandemic developed over time? Well, clearly, we learnt about social distancing, with particular concern about both aerosol and surface spread. We learnt that asymptomatic carriers can and will spread the illness. We know that people are the most infectious before they become sick, or early in the illness. The risk of severe COVID is clearly an important issue; age, obesity, smokers, who tend to have more ACE receptors, cardiovascular illness, COPD, diabetes, etc. Interestingly, though it's not related to ACE inhibitors, it's not related to inhaled corticosteroid use. So, we really shouldn't change anything in terms of prevention, and actually the best thing to do is to keep patients controlled in their conditions to prevent the severe COVID. Lastly, we have vaccinations, which are not quite being done as frequently or as comprehensively across the population as they should be yet, but that's our hope for the future with respect to COVID-19. Thank you.

\section{MARCUS PETER KENNEDY}

Hi. My name is Dr. Marcus Kennedy, and I'm a consultant respiratory physician and interventional pulmonologist at Cork University Hospital Ireland. My area of interest is lung cancer and interventional pulmonology.

The first question is, how has COVID impacted my research? COVID has impacted my research. Our group has a research interest in bronchoscopy, including single-use bronchoscopy, and we were investigating this new technology prior to the COVID pandemic. The COVID pandemic has brought this technology to the forefront. There are a number of clinical scenarios where single-use flexible bronchoscopes have advantages, and reasons for this include ease of mobility, allowing bronchoscopy in the ICU, bronchoscopy in the emergency department and emergency bronchoscopy outside the health care facility, and all these indications were brought to the forefront with the COVID pandemic. These scopes are practical, allowing out-of-hours bronchoscopy, allowing bronchoscopy at the end of a day list where staff are not required to clean scopes.
These also allow weekend bronchoscopy staff who are not available to clean scopes. They are also used in specific scenarios including immunocompromised patients and a patient with prion disease, where in the past a reusable scope would have to be destroyed after being used on a patient with prion disease. They are also important for scope training, for veterinary procedures and large animal and cadaveric research. Particularly in the era of COVID, these scopes have advantages in that they don't need to be cleaned. They are completely sterile when put into the patient, and there is a concern that a reusable scope may cross-contaminate COVID from one patient to another. In patients with COVID, the advantage of not having to clean a scope would reduce the risk of contamination from the patient with COVID to the health care worker who is cleaning the scope.

The next question is, how did COVID impact your clinic during the initial outbreak? As a respiratory physician I was at the forefront of looking after sick patients with COVID. Access for other respiratory patients to services including lung cancer patients and COPD patients was clearly negatively impacted at the outset of the COVID pandemic.

How has this initial reaction to the pandemic developed over time and what lessons have been learnt? Our hospital group was at the forefront of wave 3 of COVID in Ireland from January to February 2021. Our hospital group became involved in international studies in COVID-19 treatment. Our group learned how to separate COVID and non-COVID care and thus maintain critical non-COVID services, including lung cancer.

\section{OUTRO}

That brings us to the end of our Pulmonary Therapy podcast on COVID-19 "Research and Real-World Experiences from the Editorial Board". Thank you for listening, and thanks to all of the Editorial Board members who contributed-Drs. Kai Michael Beeh, Nazia Chaudhuri, Timothy Craig, Alan Kaplan and Marcus Kennedy. Thank you very much. 


\section{ACKNOWLEDGEMENTS}

Funding. No funding or sponsorship was received for this study or publication of this article.

Authorship. All named authors meet the International Committee of Medical Journal Editors (ICMJE) criteria for authorship for this article, take responsibility for the integrity of the work as a whole, and have given their approval for this version to be published.

Disclosures. Dr. Kai-Michael Beeh and/or the institution Dr. Beeh represents have received compensation for services on advisory boards or consulting for AstraZeneca, Berlin Chemie, Boehringer, Chiesi, GSK, Novartis, Pohl Boskamp, TEVA and sterna, and compensation for speaker activities in scientific meetings supported by AstraZeneca, Berlin Chemie, Boehringer, Cytos, ERT, GSK, Novartis, Pfizer, Pohl Boskamp, and TEVA. The institution has further received compensation for design and performance of clinical trials from AstraZeneca, Boehringer, GSK, Novartis, Parexel, Pearl Therapeutics, and sterna. Nazia Chaudhuri has received research grants from $\mathrm{BI}$ and Roche and has done consultancy work for BI, Roche, Novartis and Redex. Timothy Craig declares the following: Research-Regeneron, GSK, Grifols, CSL Behring, Takeda, BioMarin, Astra Zeneca, Biocryst. Speaking-Regeneron, Grifols, CSL Behring, Biocryst, Takeda. Consultant- Spark, BioMarin, Novartis, Biocryst, Pharming, Takeda, Grifols, GKK. Alan Kaplan has received support as a speaker or Advisory Board member for AstraZeneca, Behring, Boehringer Ingelheim, Covis, Griffols, GSK, Novo Nordisk, Pfizer,
Sanofi, Teva and Trudell Medical. Marcus Peter Kennedy has received educational grants for purchasing simulators and other training equipment valued 1-10000 Euro from The Surgical Company, Cook Medical, Menarini Pharmaceuticals and Orion Pharmaceuticals. He also has a non-disclosure contract and current grant applications with Boston Scientific.

Compliance with Ethics Guidelines. This article is based on previously conducted studies and does not contain any studies with humanparticipants or animals performed by any of the authors.

Data Availability. Data sharing is not applicable to this article as no datasets were generated or analyzed during the current study.

Open Access. This article is licensed under a Creative Commons Attribution-NonCommercial 4.0 International License, which permits any non-commercial use, sharing, adaptation, distribution and reproduction in any medium or format, as long as you give appropriate credit to the original author(s) and the source, provide a link to the Creative Commons licence, and indicate if changes were made. The images or other third party material in this article are included in the article's Creative Commons licence, unless indicated otherwise in a credit line to the material. If material is not included in the article's Creative Commons licence and your intended use is not permitted by statutory regulation or exceeds the permitted use, you will need to obtain permission directly from the copyright holder. To view a copy of this licence, visit http://creativecommons.org/licenses/bync/4.0/. 